\title{
ACTUALIDAD DE LA ANEMIA INFANTIL EN LA REGIÓN ICA
}

\author{
NEWS OF CHILDHOOD ANEMIA IN THE ICA REGION
}

\begin{abstract}
Alex Huamán-Navarro ${ }^{1,2, a}$.
1. Escuela Profesional de Medicina Humana, Universidad Privada San Juan Bautista, Ica, Perú.

2. Asociación de Investigación y Ciencia de los Estudiantes de Medicina de la Universidad Privada

San Juan Bautista (ASICEM UPSJB), Ica, Perú.

a. Estudiante de Medicina Humana.
\end{abstract}

DOI: https://doi.org/10.35563/rmp.v9i1.303

Correspondencia:

Alex Stiven Huamán Navarro

Dirección: Av. Montevideo 249 - La

Tinguiña, Ica, Perú.

Número de celular:

(+51) 944916960

Correo Electrónico:

alex_hn22@outlook.com

\section{Como Citar}

Huamán-Navarro A. Actualidad de la anemia infantil en la Región Ica. Rev méd panacea. 2020;9(1): 65-66. doi: https://doi.org/10.35563/rmp.v9i1.303

Recibido: 17 - 08 - 2019

Aceptado: 20 - $09-2019$

Publicado: 22 - 09 - 2019

\section{Sr. Editor:}

La anemia es considerada a nivel mundial como un problema de salud pública, las mujeres en la edad fértil, las embarazadas y los niños menores de 5 años son un grupo especial con mayor riesgo de desarrollarla (1). En el Perú, actualmente, la anemia infantil afecta al $43,6 \%$ de los niños y niñas menores de 36 meses de edad, lo que significa que cerca de 743 mil niños menores de 3 años padecen esta afección (2).

En el departamento de Ica, el 43,1\% de niños y niñas entre 6 y 35 meses de edad tienen anemia (3), que, si bien es cierto este se encuentra mínimamente por debajo del promedio nacional, no excluye que no siga siendo un problema de salud pública para la región. Según el último reporte del Sistema de Información de Indicadores de Estado Nutricional (SIEN), en lo que va del año, la provincia de Chincha es la que refleja un mayor porcentaje de anemia en niños menores de 5 años (33,86\%), y la que menos expresa anemia en ese rango de edad es Pisco (12,28\%)(4)(gráfico 1).

Gráfico 1. Porcentaje de anemia por provincias en niños menores de 5 años en la región Ica

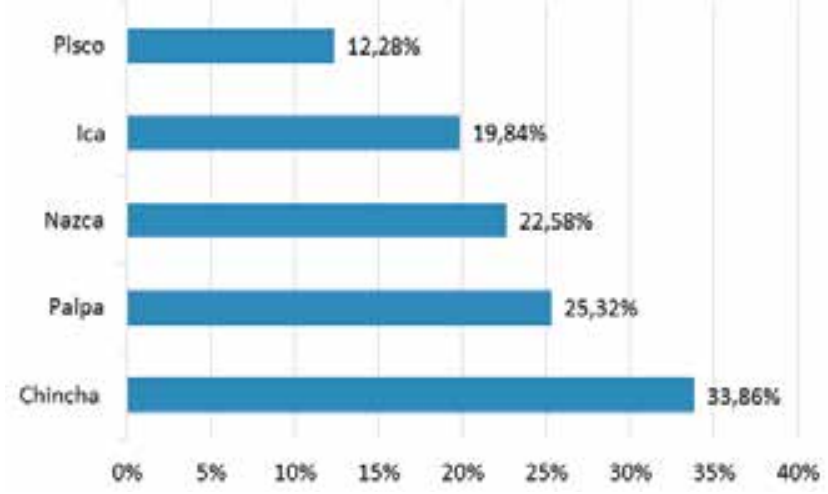

Fuente: SIEN - Dirección Regional de Salud de Ica, hasta Julio del 2019.

SIEN: Sistema de Información de Indicadores de Estado Nutricional.

Si consideramos que la anemia afecta el desarrollo neurológico y psicomotor, y que este proceso es fundamental en los primeros años de vida (5), entonces en realidad estaríamos poniendo en riesgo no solo la salud mental y psicológica, sino también el desarrollo del aprendizaje de los niños y niñas de nuestra región.

La anemia también afecta la conducta socioemocional de aquellos que la padecen, especialmente si esta se desarrolla a temprana edad, pues hay estudios que demuestran que los niños que desarrollaban anemia, en los años posteriores mostraraban conductas de respuestas adversas y que más adelante esto les podría generar conflicto en su entorno familiar y social (5).

Por ello, es importante intervenir, evaluar y buscar mejores estrategias de prevención y/o adherencia de los programas hacia esta población tan vulnerable, para tratar de precaver las consecuencias importantes que puedan desarrollar. Estos datos nos podrían ayudar a investigar en un futuro un poco más acerca de las causas del constante porcentaje de esta afección en la región Ica, para poder así conseguir las metas establecidas hacia el 2021. 


\section{REFERENCIAS BIBLIOGRÁFICAS}

1. Lopez A, Cacoub P, Macdougall IC, Peyrin-Biroulet L. Iron deficiency anaemia. Lancet (London, England) [Internet]. 2016 Feb 27 [cited 2019 Aug 22];387(10021):907-16. Available from: http://www.ncbi.nlm.nih.gov/pubmed/26314490

2. Ministerio de Desarrollo e Inclusión Social. Plan multisectorial de lucha contra la anemia [Internet]. Lima; 2018 [cited 2019 Aug 22]. Available from: http://www.midis.gob.pe/dmdocuments/plan-multise ctorial-de-lucha-contra-la-anemia-v3.pdf

3. Dirección General de Seguimiento y Evaluación. Reporte Regional de indicadores sociales del departamento de Ica [Internet]. Lima; 2019 [cited 2019 Aug 25]. Available from:

http://sdv.midis.gob.pe/redinforma/Temp/ICA.pdf
4. DIRESA ICA. Sistema de Información de indicadores de estado nutricional [Internet]. DIRESA. Ica; 2019 [cited 2019 Aug 25]. p. 4. Available from: https://app.powerbi.com/view?r=eyJrljoiNDk2ZWRI ODAtMjU5Mi00ZmEyLTIkMjltYWYxMmNkYWU5N2 M2liwidCI6ljgxZTcwMWQ1LTBkNzAtNGZkZC05Mzg 4LWM4YmE5ZDdhOTk3NSIsImMiOjR9

5. Zavaleta N, Astete-Robilliard L. Efecto de la anemia en el desarrollo infantil: consecuencias a largo plazo. Rev Peru Med Exp Salud Publica [Internet]. 2017 [cited 2019 Aug 31];34:716-22. Available from: https://www.scielosp.org/article/rpmesp/2017.v34n 4/716-722/

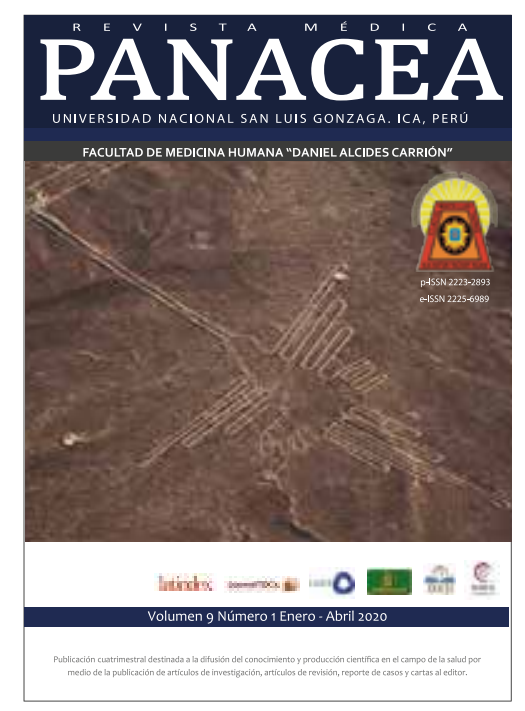

